\title{
Diagnostic Screening of Colon Cancer Through Quantification of MicroRNAs by Absolute Digital(d) PCR in Human Stool
}

\section{ISSN: 2640-9208}

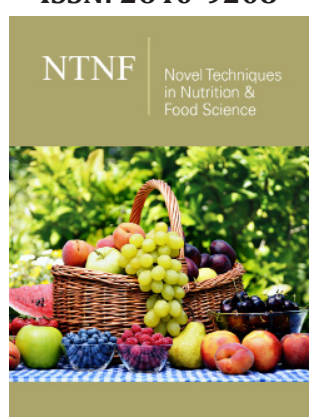

${ }^{* 1}$ Corresponding author: Farid Ahmed E, Institute for Research in Biotechnology, USA

Submission: 翉 November 04, 2019

Published: 洯 November 07, 2019

Volume 4 - Issue 4

How to cite this article: Farid Ahmed E. Diagnostic Screening of Colon Cancer Through Quantification of MicroRNAs by Absolute Digital(d) PCR in Human Stool. Nov Tech Nutri Food Sci. 4(4) NTNF.000592.2019.

DOI: 10.31031/NTNF.2019.04.000592

Copyright@ Farid Ahmed E. This article is distributed under the terms of the Creative Commons Attribution 4.0 International License, which permits unrestricted use and redistribution provided that the original author and source are credited.

\section{Farid Ahmed E*}

Institute for Research in Biotechnology, USA

\section{Opinion}

No validated micro(mi) RNA diagnostic test currently exist for the diagnostic screening of colon cancer (CC). MiRNAs are good candidates for markers for cancer diagnosis, prognosis or response to therapy. Profiles of miRNA expression differ between normal tissues and tumor types, and evidence suggests that miRNA expression profiles cluster similar tumor types together more accurately than expression profiles of protein-coding mRNA genes. A small number of miRNAs can differentiate cancer from normal, and miRNAs in stool remain mostly intact for detection We first carried a microarray study on immunocaptured stool colonocytes on 15 subjects and selected a panel of 14 preferentially expressed mature miRNAs associated with colon cancer (12 Up-Regulated, miR-19a, miR-20a, miR-21, miR-31, miR-34a, miR-96, miR-106a, miR-133a, miR-135b, miR-206, miR-224 and miR-302; and 2 Down-Regulated, miR-143 and miR-145). We then used absolute quantitative digital PCR on these 15 stool samples from stages 0-4 using a chip based Absolute Quant Studio ${ }^{\mathrm{TM}}$ 3D Digital PCR Applied Biosystem 3D instrument, using 96 well plates.

To ensure that we have chosen human and not bacterial small total RNA, we have carried out co-extraction protocols with Escherichia coli K1 strain RS18, Agilent electrophoretic patterns, and sequenced random samples throughout this research using mRNA/miRNA sequencing. The miRNA expression data are first analyzed with parametric statistics such as Student $t$-test or analysis of variance (ANOVA) test if the data distribution is random, or with nonparametric Kruskall-Wallis, Mann-Whitney and Fisher exact tests if the distribution is not random. If necessary, more complicated models such as multivariate analysis and logistic discrimination are employed. For the corrected index, cross-validation is used to protect against overfitting. Digital PCR is a new approach to miRNAs quantification that offers alternate method to qPCR for absolute quantification, by partitioning a sample of DNA or cDNA into many individual, parallel PCR reactions; some of these reactions contain the target molecule (positive), while others do not (negative). A single molecule can be amplified>million-fold.

During amplification, TaqMan chemistry with dye-labeled probes is used to detect sequence-specific targets. If no target sequence is present, no signal accumulates. Following PCR, the fraction of negative reactions is used to generate an absolute count of the number of sample's target molecules, without standards or endogenous controls. Our quantitative DPCR miRNA data showed that the quantitative changes in the expression of a few mature miRNA genes in stool, associated with right and left colon cancer, provides for a more convenient, sensitive and specific diagnostic screening markers, more useful than test markers currently available on the market, such as the low-sensitivity $(<15 \%)$ fecal occult blood test (FOBT); result in better compliance; and is more economical than the invasive/expensive colonoscopy exam in colon cancer (CC). Initial test performance characteristics of the miRNA approach showed that the test has a high numerical predictive value in colon cancer. Moreover, underpinning of the miRNA markers as a function of total RNA showed that the test can numerically differentiate between control and CC, particularly at the early stages of that cancer. 
Innovation of the DPCR-miRNA stool screening approach lies in the collective use of many methods such as: immune paramagnetic beads to capture colonocytes from the complicated stool environment, whose fragile total small RNA is stabilized immediately after stool shedding by commercially-available preparations kits so it does not fragment, followed by standardized analytical quantitative miRNA DPCR-chip profiling in noninvasive stool samples, which are neither labor intensive, nor require extensive sample preparation, to develop a panel of few stable miRNAs for absolute quantitative diagnostic quantification of early CC, cheaply and with more sensitivity/specificity than available commercial CC tests We propose to extend our results to a larger prospective and randomized five-years nested case-control epidemiological study, to validate the expression of the above 14 miRNAs, in stool of 180 individuals in an epidemiologically designed randomized study. Fewer, or even one miRNA, may suffice to serve as an efficient and a quantitative marker for the noninvasive diagnostic screening of CC in human stool. Although we may miss exosome RNA, a parallel test could also be carried out on miRNAs obtained from stool samples to compare the extent of loss when colonocytes are only used, and an appropriate corrections for exosome loss can then be made The above approach when combined with bioinformatics' analysis, to correlate miRNA seed data with our previously published messenger (m)RNA target data in stool, allows for a thorough mechanistic understanding of how miRNA genes regulate mRNA expression.

For CC screening, miRNA markers are much more comprehensive and preferable to a DNA-, epigenetic-, mRNA- or a protein-based marker. An added advantage of use of the stable, nondegradable miRNAs by PCR expression chip-based methods is automation, making it more economical and easily acceptable by lab personnel. Data showed that the quantitative changes in the expression of a few mature miRNA genes in stool, associated with right and left CC, gives more sensitive \& specific diagnostic screening markers than tests available on the market (FOBT, mutation tests), better compliance, and is more economical than the current gold standard (colonoscopy exam). Test performance characteristics showed that the test has a high numerical predictive value in $\mathrm{CC}$, and the test numerically differentiates between controls and CC at an early cancer stage. 